Editorial Letter

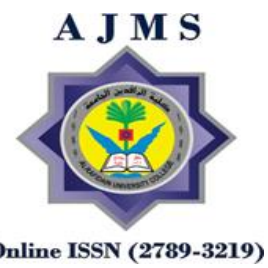

\title{
Nanobodies in the Diagnosis and Treatment of Cancer
}

\author{
Ismail I. Al-Janabi (B.Sc. M.Sc. Ph.D.) \\ Retired Academic, Freelance Consultant Pharmacist and Science Writer, Surrey, England
}

Received: August 2021; Accepted: September 2021

Corresponding author: Ismail I. Al-Janabi, Retired Academic, Freelance Consultant Pharmacist and Science Writer, Surrey KT198GY, U. K.; Email: ismail.janabi@gmail.com

Article Citation: Al-Janabi II. Nanobodies in the diagnosis and treatment of cancer. Al-Rafidain J Med Sci. 2021;1:49.

Finding good treatments for cancer remains challenging. Surgical removal of the tumor, although effective, is constrained by factors such as the site, type, and the metastatic condition of the tumor. While radiation and chemotherapy appear to be potent in eliminating cancer cells, they have the disadvantage of killing bystander cells with consequent severe side effects $[1,2]$. Over the past several years, the use of therapies where a specific protein is targeted has gained considerable momentum [1]. A major class of these targeted therapies is monoclonal antibodies (mAbs), which are proteins specifically produced to bind to one substance. From the time they were first generated in 1975 , mAbs were quickly adopted into clinical practice, with over $30 \mathrm{mAbs}$ now approved for clinical use in cancer by the Food and Drug administration (FDA) [3]. In addition to their therapeutic use, alone or as vehicles for the targeted delivery of other molecules, mAbs are also used in Positron Emission Tomography (PET), Single Proton Emission Computerized Tomography (SPECT) and optical imaging in general to direct reagents to the required site [4]. However, the use of mAbs has been hampered by the fact that their relatively large size limits tumor penetration. Further drawbacks of mAbs have been their high production cost and the possible induction of immunogenic responses [5]. The discovery of heavy-chain only antibodies in the serum of camelids (e.g. camels, llamas and alpacas) around 30 years ago has prompted investigations into their possible use as alternatives to mAbs (and attracted much attention recently during the COVID-19 pandemic as a therapy for this disease). These heavy-chain only antibodies, and modified versions of them, are now called nanobodies (Nbs) and represent a novel and unique class of antigen-binding molecules. They possess superior properties compared to conventional monoclonal antibodies, such as small size, high stability and strong affinity for binding sites $[3,4]$. These properties make them ideal candidates for the next generation of targeted therapy and diagnostics. They are currently extensively used in research and biotechnology and are under clinical investigation for a wide variety of diseases, including cancer. More recently, Caplacizumab received approval from major world regulatory agencies for the treatment of thrombotic thrombocytopenic purpura [5].

\section{REFERENCES}

1. Baudino TA. Targeted Cancer Therapy: The Next Generation of Cancer Treatment. Curr Drug Discov Technol.2015;12(1):320.

2. Hu Y, Liu C, Muyldermans S. Nanobody-based delivery systems for diagnosis and targeted tumor therapy. Front Immunol. 2017;8:1442.

3. Yang EY, Shah K. Nanobodies: Next generation of cancer diagnostics and therapeutics. Front Oncol. 2020;10:1182.

4. Kang W, Ding C, Zheng D, Ma X, Yi L, Tong X, et al. Nanobody conjugates for targeted cancer therapy and imaging. Technol Cancer Res Treat. 2021;20:15330338211010117.

5. Jovčevska I, Muyldermans S. The therapeutic potential of nanobodies. BioDrugs. 2020;34(1):11-26. 